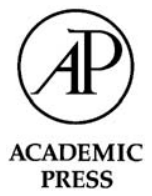

\title{
Expected utility theory without the completeness axiom
}

\author{
Juan Dubra, ${ }^{\mathrm{a}}$ Fabio Maccheroni, ${ }^{\mathrm{b}}$ and Efe A. Ok ${ }^{\mathrm{c}, *}$

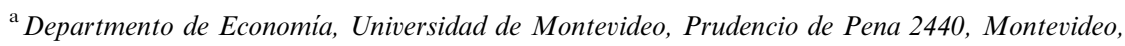 \\ CP 11600, Uruguay \\ ${ }^{\mathrm{b}}$ Istituto di Metodi Quantitativi, Università Bocconi, viale Isonzo 25, 20135 Milano, Italy \\ ${ }^{\mathrm{c}}$ Department of Economics, New York University, 269 Mercer St., 7th Floor, New York, NY 10003, USA
}

Received 13 December 2001; final version received 13 February 2003

\begin{abstract}
We study the problem of obtaining an expected utility representation for a potentially incomplete preference relation over lotteries by means of a set of von Neumann-Morgenstern utility functions. It is shown that, when the prize space is a compact metric space, a preference relation admits such a multi-utility representation provided that it satisfies the standard axioms of expected utility theory. Moreover, the representing set of utilities is unique in a welldefined sense.

(C) 2003 Elsevier Science (USA). All rights reserved.
\end{abstract}

JEL classification: D11; D81

Keywords: Incomplete preference relations; Expected utility

\section{Introduction}

The von Neumann-Morgenstern expected utility theorem is one of the most fundamental results of the theory of individual decision making. It shows that a preference relation defined on a lottery space has an expected utility representation, provided that it is a complete and transitive binary relation that satisfies the standard independence and continuity axioms. Given the importance of this result, it is not

\footnotetext{
${ }^{*}$ Corresponding author.

E-mail addresses: dubraj@um.edu.uy (J. Dubra), fabio.maccheroni@uni-bocconi.it (F. Maccheroni), efe.ok@nyu.edu (E.A. Ok).
} 
surprising that there is a large number of studies that investigate its alterations which arise due to the relaxation of its various postulates. However, only few of these studies focus on the completeness assumption; it is presently not known if there is a reasonable way of modifying the expected utility theorem to include incomplete preferences within its coverage. Our objective here is to offer this sort of a modification, and to prove an expected utility theorem for incomplete preference relations defined over lotteries.

Before stating the nature of our contribution more concretely, let us note that there are several economic reasons why one would like to study incomplete preference relations. First of all, as advanced by several authors in the literature, it is not evident if completeness is a fundamental rationality tenet the way the transitivity property is. Aumann [2], Bewley [3] and Mandler [14], among others, defend this position strongly from both the normative and positive viewpoints, and suggest that a preference relation should in fact be considered as a potentially incomplete preorder, thereby allowing for the occasional "indecisiveness" of the agents. ${ }^{1}$ Secondly, there are economic instances in which a decision maker is in fact composed of several agents each with a possibly distinct objective function. In such a situation requiring the completeness of the preferences of the decision maker may be too demanding. The same reasoning applies to social choice problems; after all, the Pareto dominance is a commonly used social welfare ordering (as in the first and second fundamental welfare theorems), while it is an incomplete preorder.

Since these issues are discussed at length in the literature, we shall not discuss the potential importance of incomplete preferences for economic modeling at large, but rather proceed to discuss how one may handle the problem of actually representing such preferences. $^{2}$ Curiously, the basic idea has already been suggested, albeit elusively, by von Neumann and Morgenstern [26, pp. 19-20]:

... We have conceded that one may doubt whether a person can always decide which of two alternatives ... he prefers. If the general comparability assumption is not made, a mathematical theory ... is still possible. It leads to what may be described as a many-dimensional vector concept of utility. This is a more complicated and less satisfactory set-up, but we do not propose to treat it systematically at this time.

\footnotetext{
${ }^{1}$ In concert with this position, it is recently shown that incomplete preferences can also be "derived" from choices by means of a standard revealed preference exercise, provided that Houthakker's axiom of revealed preference is suitably relaxed; see [7,20]. Similarly, Danan [5] provides an alternative foundation for incomplete preferences in a dynamic choice setup that allows for incomplete knowledge of one's future tastes. Finally, Masatlioglu and Ok [15] shows how incompleteness of preferences may arise due to status quo bias and/or endowment effect, again within the revealed preference paradigm.

${ }^{2}$ A closely related issue was studied by Aumann [2] and Kannai [11]. These authors were interested in finding an extension of an incomplete preference relation, defined over lotteries, that admits an expected utility representation. Unfortunately, as also noted by Majumdar and Sen [13], this approach falls short of yielding a representation theorem, for it does not characterize the preference relations under consideration. More on this in Section 3.2.
} 
In evaluation of this statement, Aumann [2, p. 449] notes that “... Details were never published. What they probably had in mind was some kind of mapping from the space of lotteries to a canonical partially ordered euclidean space, ... but it is not clear to me how this approach can be worked out." Our objective here is actually nothing other than formalizing Aumann's interpretation of the von NeumannMorgenstern suggestion.

To make things a bit more precise, let us denote by $X$ the set of certain prizes, and consider a preference relation $\succsim$ which is defined as a (potentially incomplete) preorder on the set of all lotteries on $X$. It is obvious that one cannot represent $\succsim$ in the standard way by using a single von Neumann-Morgenstern utility function, if $\succsim$ is actually incomplete. But one may do so by means of a set of utility functions defined on $X$. Thus the representation notion we suggest requires one to come up with a set $\mathscr{U}$ of real functions on $X$ such that, for all lotteries $p$ and $q$,

$$
p \succsim q \text { if and only if } \mathbf{E}_{p}(u) \geqslant \mathbf{E}_{q}(u) \text { for all } u \in \mathscr{U},
$$

where $\mathbf{E}_{r}(u)$ stands for the expectation of $u$ with respect to the lottery $r=p, q$. We are, then, interested in obtaining an expected multi-utility representation for incomplete preference relations. This seems to correspond well to the intuition indicated in the von Neumann-Morgenstern and Aumann quotations given above.

A close relative of the above representation concept is actually suggested also by Shapley and Baucells [24] (see Remark 3 below), and is studied in the context of utility theory under certainty by Ok [21]. This concept clearly carries a stochastic dominance flavor, and hence brings the expected utility theory one step closer to the theory of stochastic orders. ${ }^{3}$ More generally, this particular formulation of utility representation ties the expected utility theory to the theory of multi-objective decision making. While this link is often suggested to motivate the study of incomplete preferences, an axiomatization of the representation we suggest here will clearly make the connection a concrete one. What is more, such an axiomatization sheds light into the role of the completeness assumption in the classical expected utility theorem. For all practical purposes, our approach shows precisely how this theorem changes in the absence of the completeness axiom.

Put concretely, we focus in this paper on the case in which $X$ is a compact metric space, and prove that the standard independence axiom and a mild strengthening of the standard continuity property suffice to yield an expected multi-utility representation in terms of continuous utility functions. In what follows, we shall also determine in what sense such a representation may be regarded as unique, show how it can be strengthened in the case of monetary lotteries, demonstrate that it can be used to complete a preference relation in the sense of [2], and discuss the potential difficulties in extending the present approach to a more general class of prize spaces.

\footnotetext{
${ }^{3}$ In fact, the preorders that admit such a vector-valued representation are called integral stochastic orders (see [27]), and have been studied extensively in the literature on applied probability; see, inter alia, [18] - which is an annotated bibliography - [19,23]. To the best of our knowledge, however, the integral stochastic orders are so far not investigated axiomatically.
} 


\section{Expected multi-utility representation}

\subsection{Preliminaries}

We take an arbitrary compact metric space $X$ as the set of all certain prizes (degenerate lotteries), and let $C(X)$ stand for the set of all continuous real maps on $X$ (which is topologized by the sup-norm), respectively. The set of all Borel probability measures (lotteries) over $X$, endowed with the topology of weak convergence, is denoted by $\mathscr{P}(X){ }^{4}$

We define a preference relation as any reflexive and transitive binary relation on $\mathscr{P}(X)$. This should be contrasted with the standard theory in which a preference relation is almost exclusively assumed also to be complete. To stress this point, we note that the first-order stochastic dominance ordering (defined on a compact subset of $\mathbf{R}$ ) is a preference relation in the general sense of the term adopted here, while this is not the case for much of the standard theory.

The most fundamental postulate of the expected utility theory is of course the independence axiom which is stated formally as follows.

Independence axiom. For any $p, q, r \in \mathscr{P}(X)$ and any $\lambda \in(0,1)$,

$p \succsim q$ implies $\lambda p+(1-\lambda) r \succsim \lambda q+(1-\lambda) r$.

The independence axiom is coupled in the standard theory either with an (ordertheoretic) Archimedean axiom or with a form of (topological) continuity property. We will follow the latter approach in this paper. In particular, we shall concentrate on the following two continuity conditions.

Weak continuity axiom. $\{\lambda \in[0,1]: \lambda p+(1-\lambda) r \succsim \lambda q+(1-\lambda) s\}$ is closed in $[0,1]$ for any $p, q, r, s \in \mathscr{P}(X)$.

Continuity axiom..$^{5}$ For any convergent sequences $\left(p_{n}\right)$ and $\left(q_{n}\right)$ in $\mathscr{P}(X)$,

$p_{n} \succsim q_{n}$ for all $n$ imply $\lim p_{n} \succsim \lim q_{n}$.

While Shapley and Baucells [24] adopt the weak continuity axiom, we will work here with the (stronger) continuity axiom. In fact, it is an open problem to determine if the continuity axiom can be replaced with weak continuity in our main representation theorem. However, there is at least one noteworthy case in which these two continuity requirements are in fact equivalent, namely, the case of finite

\footnotetext{
${ }^{4}$ For concreteness, we recall that this topology is metrizable, and a sequence $\left(p_{n}\right)$ in $\mathscr{P}(X)$ converges to $p \in \mathscr{P}(X)$ relative to this topology if and only if $\int_{X} f d p_{n} \rightarrow \int_{X} f d p$ for all $f \in C(X)$.

${ }^{5}$ We should also make note of the following more common version of this axiom [10]: For all $q \in \mathscr{P}(X)$, the sets $\{p: p \succsim q\}$ and $\{p: q \succsim p\}$ are closed in $\mathscr{P}(X)$. We do not know if the main theorem of this paper can be proved with this weaker continuity condition, except in the case where $X$ is a finite set. Conceptually speaking, however, we do not see much difference between these two continuity conditions. In fact, some textbooks (such as Mas-Colell et al. [16, p. 46]) "define" the continuity axiom for an arbitrary preference relation on a topological space precisely as we do here.
} 
prize spaces. This is the content of the next proposition whose proof appears in Section 3 along with the proofs of our main results.

Proposition 1. Let $X$ be a nonempty finite set and $\succsim$ a preference relation on $\mathscr{P}(X)$ that satisfies the independence axiom. Then, $\succsim$ satisfies the continuity axiom if and only if it satisfies the weak continuity axiom.

As noted above, we do not know to what extent one may be able to relax the finiteness assumption in this proposition. Leaving this question unanswered, we now turn to analyze the expected utility theory that can be founded on the independence and continuity axioms.

\subsection{Main results}

While the significance of incomplete preference relations is noted in the literature, a definitive expected utility representation for such preorders does not seem to be agreed upon. Given the well-known characterization of the stochastic dominance orderings in terms of linear functionals that possess an expected utility form, we would like to propose here a multi-utility representation for such a preorder. Put more precisely, we seek here a set $\mathscr{U}$ of utility functions on $X$ such that, for all $p, q \in \mathscr{P}(X)$,

$$
p \succsim q \text { if and only if } \int_{X} u d p \geqslant \int_{X} u d q \text { for all } u \in \mathscr{U} \text {. }
$$

This representation notion is certainly alluded to by $[2,11]$, and studied in $[4,6]$, albeit nonaxiomatically. It may be viewed as a dual of the theory of Knightian uncertainty. Loosely stated, in that theory, one compares horse race lotteries by means of taking expectations of a single utility function with respect to a set of probability measures [3], whereas in our setting of objective uncertainty, the roulette lotteries are compared by taking expectations of a set of utility functions with respect to the given (objective) lotteries. ${ }^{6}$

The main result of this paper states that any preference relation that satisfies the independence and continuity axioms admits an expected multi-utility representation, provided that the prize space $X$ is compact. ${ }^{7}$ Moreover, when $X$ is finite, the

\footnotetext{
${ }^{6}$ One can, of course, aggregate the resulting set of expectations in various ways, may this set be arising from nonunique priors or multiple utility functions. Gilboa and Schmeidler [9], for instance, develops a decision theory in which a single utility value is assigned to each horse race lottery by taking the minimum of this set, and dually, Maccheroni [12] shows that a particular relaxation of the independence axiom in the von Neumann-Morgenstern setup would yield a set of utilities the expectations of which are again aggregated by means of the min operator. The spirit of these two papers is, however, quite distinct from the present one as they deal with complete preference relations.

${ }^{7}$ A similar result in an Anscombe-Aumann environment with a finite state space and countable sureoutcomes is given by Seidenfeld et al. [22] who obtain an expected state-dependent multi-utility representation. However, the continuity axiom used in [22] is far from standard.
} 
continuity axiom can be replaced in this statement with the weak continuity axiom (Proposition 1).

Expected multi-utility theorem. Let $X$ be a compact metric space, and let $\succsim$ be a binary relation on $\mathscr{P}(X)$. $\succsim$ is a preference relation that satisfies the independence and continuity axioms if and only if there exists a closed and convex set $\mathscr{U} \subseteq C(X)$ such that (1) holds for each $p, q \in \mathscr{P}(X)$.

We now turn to generalize the uniqueness part of the classic expected utility theorem in our multi-utility context. This generalization can in fact be carried out in an arbitrary (not necessarily compact) metric space $X$, provided that the utility functions are chosen from $C_{b}(X)$, the set of all continuous and bounded real functions on $X$. The upshot is that if the sets $\mathscr{U}$ and $\mathscr{V}$ in $C_{b}(X)$ represent a preference relation $\gtrsim$ as in (1), then $\mathscr{V}$ must belong to the closed convex cone generated by $\mathscr{U}$ and all constant functions; this is the content of the forthcoming uniqueness theorem. , $^{8}$

Clearly, a special case of this observation is the standard uniqueness result of expected utility theory, for the closure of the convex cone generated by a singleton $\{u\}$ and all the constant functions is none other than the set $\left\{a u+b:(a, b) \in \mathbf{R}_{+} \times \mathbf{R}\right\}$. To give another example, let us note that, for any subset $\mathscr{U}$ of $C_{b}(X)$ and any $(a, b) \in \mathbf{R}_{++} \times \mathbf{R}$, the sets $\mathscr{U}$ and $a \mathscr{U}+b$ represent the same preference relation as in (1). However, there are sets that are not of this form but that still represent the same preference relation. For example, let $X:=[0,1]$ and $\mathscr{U}:=\{u, v\}$ where $u, v \in C(X)$ are defined as $u(x):=x$ and $v(x):=x^{2}$. Then $\mathscr{U}$ and $\mathscr{V}:=\{\alpha u+(1-\alpha) v: 0<\alpha<1\}$ represent the same preference relation on $\mathscr{P}(X)$, but $\mathscr{V}$ cannot be written as $a \mathscr{U}+b$ for any $(a, b) \in \mathbf{R}_{+} \times \mathbf{R}$.

To state formally our general uniqueness result on the set-valued expected utility representations, we define the operator $\langle\cdot\rangle: 2^{C_{b}(X)} \rightarrow 2^{C_{b}(X)}$ as

$$
\langle\mathscr{U}\rangle:=\operatorname{cl}\left(\operatorname{cone}(\mathscr{U})+\left\{\theta \mathbf{1}_{X}\right\}_{\theta \in \mathbf{R}}\right),
$$

where the closure operator is applied with respect to the weak topology on $C_{b}(X)$ (or equivalently, with respect to the sup-norm topology when $X$ is compact). ${ }^{10}$ It is easy to verify that if $\mathscr{U}$ represents $\succsim$, then so does $\langle\mathscr{U}\rangle$. The following result tells us further that $\langle\mathscr{U}\rangle$ is in fact the largest set of utility functions in $C_{b}(X)$ that represents

\footnotetext{
${ }^{8} \mathrm{~A}$ number of versions and special cases of this result have actually been noted elsewhere in the literature; see, for instance, [4,6,19].

${ }^{9}$ In this paper by a convex cone (in any vector space) we mean a nonempty convex set that is closed under nonnegative scalar multiplication. For any set $A$, cone $(A)$ stands for the smallest convex cone that contains $A$.

${ }^{10}$ We recall that the weak topology on $C_{b}(X)$ is the weakest topology on $C_{b}(X)$ that declares continuous all functionals of the form $f \mapsto \int_{X} f d \mu$ where $\mu$ is any finite Borel signed measure on $X$.
} 
$\succsim$ as in (1). This observation can be viewed as a general uniqueness theorem for expected multi-utility representations.

Uniqueness theorem. Let $X$ be a metric space. Two nonempty sets $\mathscr{U}$ and $\mathscr{V}$ in $C_{b}(X)$ satisfy

$$
\begin{aligned}
\int_{X} u d p \geqslant & \int_{X} u d q \text { for all } u \in \mathscr{U} \text { if and only if } \\
& \int_{X} v d p \geqslant \int_{X} v d q \text { for all } v \in \mathscr{V}
\end{aligned}
$$

(for each $p, q \in \mathscr{P}(X)$ ) if and only if $\langle\mathscr{U}\rangle=\langle\mathscr{V}\rangle$.

In the next section we will provide a few implications of these results, and discuss further some of the previous related work.

\section{Supplementaries}

\subsection{Preferences over monetary lotteries}

An interesting special case of the present setup is the case of monetary lotteries where $X$ is a compact interval in the real line, say, $X=[0,1]$. Since in this case we wish to incorporate the idea that "more money is preferred to less," one should examine the structure of the preference relations $\gtrsim$ on $\mathscr{P}[0,1]$ such that $p \succ_{\text {FSD }} q$ implies $p \succ q$ for all $p, q \in \mathscr{P}[0,1]$, where $\succ_{\text {FSD }}$ is the irreflexive part of the first-order stochastic dominance relation $\succsim_{\text {FSD }}$ on $\mathscr{P}[0,1]$. The issue is then to determine the structure of preferences that satisfy not only the axioms of independence and continuity, but also this monotonicity condition. To do this, let us agree to call a set $\mathscr{U}$ in $\mathbf{R}^{[0,1]}$ strictly increasing, if each $u \in \mathscr{U}$ is weakly increasing, and if $0 \leqslant a<b \leqslant 1$ implies $u(a)<u(b)$ for some $u \in \mathscr{U}$. The following is a useful corollary of our main representation theorem.

Proposition 2. Let $\succsim$ be a binary relation on $\mathscr{P}[0,1]$. $\succsim$ is a preference relation that satisfies the independence, continuity and monotonicity axioms if, and only if, there exists a strictly increasing closed and convex set $\mathscr{U} \subseteq C[0,1]$ such that (1) holds for each $p, q \in \mathscr{P}[0,1]$.

Given the general expected multi-utility theorem we have stated above, it is easy to check that any set $\mathscr{U}$ of utilities representing a monotonic preference relation $\succsim$ must be strictly increasing. Thus all we need to do here is to verify the monotonicity of a preference relation $\succsim$ for which there exists a strictly increasing $\mathscr{U}$ in $C[0,1]$ such that (1) holds for each $p, q \in \mathscr{P}[0,1]$. Take any $p, q \in \mathscr{P}[0,1]$ with $p \succ_{\text {FSD }} q$. Then $F_{p}^{-1}>F_{q}^{-1}$, that is, $F_{p}^{-1}(s) \geqslant F_{q}^{-1}(s)$ for all $s \in(0,1)$ and $F_{p}^{-1}\left(s^{*}\right)>F_{q}^{-1}\left(s^{*}\right)$ for some 
$s^{*} \in(0,1) .{ }^{11}$ Since $\mathscr{U}$ is strictly increasing, there exists a $u^{*} \in \mathscr{U}$ such that $u^{*}\left(F_{p}^{-1}\left(s^{*}\right)\right)>u^{*}\left(F_{q}^{-1}\left(s^{*}\right)\right)$, so that $u^{*} \circ F_{p}^{-1}>u^{*} \circ F_{q}^{-1}$. But $u^{*} \circ F_{p}^{-1}$ and $u^{*} \circ F_{q}^{-1}$ are left continuous, and hence

$$
\int_{[0,1]} u^{*} d p=\int_{0}^{1} u^{*}\left(F_{p}^{-1}(s)\right) d s>\int_{0}^{1} u^{*}\left(F_{q}^{-1}(s)\right) d s=\int_{[0,1]} u^{*} d q .
$$

Moreover (since $\mathscr{U}$ consists of increasing functions), $\int_{[0,1]} u d p \geqslant \int_{[0,1]} u d q$ for all $u \in \mathscr{U}$. Hence we may conclude that $p \succ q$, which proves Proposition 2.

\subsection{The extension of incomplete von Neumann-Morgenstern preferences}

As noted in the Introduction, earlier studies on relaxing the completeness axiom within the paradigm of expected utility have focused on the problem of extending a preference relation that satisfies the independence and (various forms of) the continuity axioms in such a way that the extended relation admits a von NeumannMorgenstern representation. The important work of Aumann [2], in particular, is geared towards finding a function $u: X \rightarrow \mathbf{R}$, referred to as an Aumann utility below, such that

$$
p \succ(\sim) q \text { implies } \int_{X} u d p>(=) \int_{X} u d q
$$

for all $p, q \in \mathscr{P}(X)$. A major disadvantage of this approach is that one cannot recover the preference relation $\succsim$ from its Aumann utility. So, in contrast to $\mathscr{U}$ in (1), the information contained in an Aumann utility for $\succsim$ is strictly less than $\succsim$. Maximization of an expected Aumann utility on a given constraint set $S$ leads to a $\succsim$-maximal element in $S$, whereas the vector-maximization of all expected members of $\mathscr{U}$ leads to the set of all $\succsim$-maximal elements in $S$.

It is, however, still worth knowing if an Aumann utility exists in the present context. Fortunately, mostly because we work with a continuity condition stronger than that adopted by Aumann, the answer is yes. ${ }^{12}$

Proposition 3. Let $X$ be a compact metric space, and let $\succsim$ be a preference relation on $\mathscr{P}(X)$. If $\succsim$ satisfies the independence and continuity axioms, then it possesses a continuous Aumann utility.

\footnotetext{
${ }^{11}$ The pseudoinverse distribution function of a probability measure $p \in \mathscr{P}[0,1]$ is defined by $F_{p}^{-1}(s):=$ $\min \{t \in[0,1]: p([0, t]) \geqslant s\}$ for all $s \in(0,1)$. It is easily checked to be increasing and left continuous. Moreover, pseudoinverses display these two useful features: (i) $\int_{[0,1]} u d p=\int_{0}^{1} u\left(F_{p}^{-1}(s)\right) d s$ for all $u \in C[0,1]$, and (ii) $p \succsim_{\mathrm{FSD}} q$ iff $F_{p}^{-1} \geqslant F_{q}^{-1}$.

${ }^{12}$ This is perhaps somewhat surprising, because one major message of Aumann [2] is that an expected utility theory without the completeness axiom cannot be pursued along the extension approach, when $X$ is infinite. However, since Aumann's related example does not work for a space of lotteries (it is proved in the mixture space $\mathbf{R}^{\infty}$ ), there is reason to believe that the said message is in fact overly pessimistic. What is more, with a slight strengthening of Aumann's continuity axiom (as adopted here), both the extension and the multi-utility approaches stand strong, at least in the case of lotteries defined over an arbitrary compact metric space.
} 
To prove this, we apply the expected multi-utility theorem to find a set $\mathscr{U}$ in $C(X)$ such that (1) holds for all $p, q \in \mathscr{P}(X)$. Clearly, it is without loss of generality to assume that $u \geqslant 0$ for all $u \in \mathscr{U}$. Since $X$ is compact, $C(X)$ is separable, and hence $\mathscr{U}$ is itself a separable metric space. Let $\left\{v_{1}, v_{2}, \ldots\right\}$ be a dense set in $\mathscr{U}$. It is readily verified that

$$
p \succsim q \text { if and only if } \int_{X} v_{n} d p \geqslant \int_{X} v_{n} d q \text { for all } n=1,2, \ldots
$$

Let $u_{n}:=2^{-n} \frac{v_{n}}{\left\|v_{n}\right\|+1}$ for each $n$, and observe that

$$
p \succsim q \text { if and only if } \int_{X} u_{n} d p \geqslant \int_{X} u_{n} d q \text { for all } n=1,2, \ldots
$$

Define $w:=\sum_{n=1}^{\infty} u_{n} \in C(X)$, and notice that, by the monotone convergence theorem, $\int_{X} w d r=\sum\left(\int_{X} u_{n} d r\right)$ for all $r \in \mathscr{P}(X)$. Let $p, q \in \mathscr{P}(X)$. It is obvious that $p \sim q$ implies $\int_{X} w d p=\int_{X} w d q$. On the other hand, by (2), $p \succ q$ implies that there exists a positive integer $N$ such that $\int_{X} u_{N} d p>\int_{X} u_{N} d q$ while $\int_{X} u_{n} d p \geqslant \int_{X} u_{n} d q$ for all $n$, whence

$$
\int_{X} w d p=\sum_{n=1}^{\infty}\left(\int_{X} u_{n} d p\right)>\sum_{n=1}^{\infty}\left(\int_{X} u_{n} d q\right)=\int_{X} w d q
$$

Thus, $w$ is a continuous Aumann utility for $\gtrsim$, and Proposition 3 is proved.

\subsection{The algebraic approach to multi-utility representation}

As noted in Section 1, Shapley and Baucells [24] advance a representation notion which actually admits the corresponding notion we introduced here as a special case. These authors identify conditions for a preference relation $\succsim$ on $\mathscr{P}(X)$ (actually on an arbitrary mixture space) to have a representation of the form

$$
p \succsim q \text { if and only if } T(p) \geqslant T(q) \text { for all } T \in \Omega,
$$

where $\Omega$ is a nonempty set of affine functionals on $\mathscr{P}(X)$. The approach of Shapley and Baucells contrasts with the present one in that it is algebraic as opposed to topological.

In the finite case the equivalence between the continuity and the weak continuity axioms (Proposition 1) makes it possible to derive our result from theirs. On the other hand, in the case of infinite $X$, it is not clear if and when the functionals $T$ in $\Omega$ do possess an expected-utility form. The problem is that it is not clear when each $T$ can be chosen to be continuous on $\mathscr{P}(X)$ relative to the topology of weak convergence. While this may sound like a technical concern at first, it should be noted that, without this issue being resolved, the Shapley-Baucells approach does not yield an expected utility 
theorem. ${ }^{13}$ Moreover, focusing only on simple lotteries does not solve the problem (see Remark 1 below for more on this).

Another difficulty with the Shapley-Baucells approach is that it does not function only in terms of the classical assumptions of independence and continuity, but it is, in addition, based on a crucial "properness" assumption which ensures that the cone $\{\lambda(p-q): \lambda>0$ and $p \succsim q\}$ has a nonempty algebraic interior by definition. Unfortunately, it is not at all easy to see what sort of a primitive axiom on a preference relation would support such a technical requirement.

\section{Proofs}

We begin with establishing a few basic facts that will prove useful in what follows. ${ }^{14}$

Lemma 1. Let $X$ be a metric space and $\succsim$ a preference relation on $\mathscr{P}(X)$ that satisfies the independence and weak continuity axioms. Then, for any $p, q \in \mathscr{P}(X)$, and any $\lambda \in(0,1]$,

$$
\lambda p+(1-\lambda) r \succsim \lambda q+(1-\lambda) r \quad \text { implies } p \succsim q .
$$

Proof. Let $p, q \in \mathscr{P}(X)$ and $\lambda \in(0,1]$ be such that $\lambda p+(1-\lambda) r \succsim \lambda q+(1-\lambda) r$. Let

$$
\bar{\alpha}:=\sup \{\alpha \in[0,1]: \alpha p+(1-\alpha) r \succsim \alpha q+(1-\alpha) r\} .
$$

Clearly $\bar{\alpha} \geqslant \lambda>0$. Using the weak continuity of $\gtrsim$ it is easily verified that $\bar{\alpha} p+(1-$ $\bar{\alpha}) r \succsim \bar{\alpha} q+(1-\bar{\alpha}) r$. Now set $\beta:=\frac{1}{1+\bar{\alpha}}$ and observe that the independence axiom yields

$$
\begin{aligned}
\beta(\bar{\alpha} p+(1-\bar{\alpha}) r)+(1-\beta) p & \succsim \beta(\bar{\alpha} q+(1-\bar{\alpha}) r)+(1-\beta) p \\
& =\beta(\bar{\alpha} p+(1-\bar{\alpha}) r)+(1-\beta) q \\
& \gtrsim \beta(\bar{\alpha} q+(1-\bar{\alpha}) r)+(1-\beta) q
\end{aligned}
$$

so that

$$
\frac{2 \bar{\alpha}}{1+\bar{\alpha}} p+\frac{1-\bar{\alpha}}{1+\bar{\alpha}} r \succsim \frac{2 \bar{\alpha}}{1+\bar{\alpha}} q+\frac{1-\bar{\alpha}}{1+\bar{\alpha}} r .
$$

But by definition of $\bar{\alpha}, \frac{2 \bar{\alpha}}{1+\bar{\alpha}} \leqslant \bar{\alpha}$, that is, $\bar{\alpha}^{2}-\bar{\alpha} \geqslant 0$. Since $\bar{\alpha}>0$, therefore, we have $\bar{\alpha}=1$, and hence the previous observation gives $p \succsim q$.

\footnotetext{
${ }^{13}$ To wit, linearity of a functional (on a topological linear space) does not guarantee that it has an integral representation. One needs continuity and linearity together for this, whereas it is a standard result in functional analysis that there is a linear functional defined on any infinite-dimensional normed linear space which is not continuous.

${ }^{14}$ These observations were noted first by Shapley and Baucells [24]. We include their short proofs here for completeness.
} 
Lemma 2. Let $X$ be a metric space and $\succsim$ a preference relation on $\mathscr{P}(X)$ that satisfies the independence and weak continuity axioms. Then, for any $p, q \in \mathscr{P}(X), p \succsim q$ holds if and only if there exist $a \lambda>0$ and $r, s \in \mathscr{P}(X)$ with $r \succsim s$ and $p-q=\lambda(r-s)$. Moreover, the set

$$
\mathscr{C}(\gtrsim):=\{\lambda(p-q): \lambda>0 \text { and } p \succsim q\}
$$

is a convex cone in the linear space generated by $\mathscr{P}(X)$, and we have

$$
p \succsim q \text { if and only if } p-q \in \mathscr{C}(\gtrsim)
$$

for any $p, q \in \mathscr{P}(X){ }^{15}$

Proof. Take any $\lambda>0$ and $r, s \in \mathscr{P}(X)$ such that $r \succsim s$ and $p-q=\lambda(r-s)$. Observe that the independence axiom gives

$$
\frac{1}{1+\lambda} p+\frac{\lambda}{1+\lambda} s=\frac{1}{1+\lambda} q+\frac{\lambda}{1+\lambda} r \succsim \frac{1}{1+\lambda} q+\frac{\lambda}{1+\lambda} s .
$$

Applying Lemma 1, $p \succsim q$ obtains. The converse claim follows upon setting $\lambda=1$. Moreover, while that $\mathscr{C}(\gtrsim)$ is a cone is trivial, its convexity follows from the independence axiom; we omit the routine details. The final claim is, on the other hand, an immediate consequence of the first part of this lemma.

Proposition 1 is proved next.

Proof of Proposition 1. Define $\mathscr{C}(\gtrsim)$ as in Lemma 2. To show that $\succsim$ satisfies the continuity axiom, it is enough to show that $\mathscr{C}(\gtrsim)$ is a closed subset of $\operatorname{span}(\mathscr{P}(X))$ (which equals $\mathbf{R}^{|X|}$ here). Let $s=\left(\frac{1}{|X|}, \frac{1}{|X|}, \ldots, \frac{1}{|X|}\right) \in \mathscr{P}(X)$, and note that $S:=$ $\{r \in \mathscr{P}(X): r \succsim s\}=(s+\mathscr{C}(\succsim)) \cap \mathscr{P}(X)$. Thus, if we can show that $S$ is closed in $\mathscr{P}(X)$, then it will follow that $\mathscr{C}(\succsim) \cap(\mathscr{P}(X)-s)$ is closed in $\mathscr{P}(X)-s$, and hence that $\mathscr{C}(\succsim)$ is closed in the linear space generated by $\mathscr{P}(X)-s$, whence in $\mathbf{R}^{|X|}$.

Let $\operatorname{aff}(S)$ denote the affine hull of $S$ (i.e. the smallest linear manifold that contains $S$ ). Now if $S$ is a singleton there is nothing to prove, so we assume that $|S| \geqslant 2$. But the independence axiom implies that $S$ is convex, so given that $\operatorname{aff}(S)$ is finite-dimensional, the relative interior of $S$ (i.e. its interior in $\operatorname{aff}(S)$ ) is nonempty; pick any $p$ in this set.

Now let $q$ be any element of the boundary of $S$ in $\operatorname{aff}(S)$, and $B$ the open unit ball in the linear space $\operatorname{aff}(S)-q$. We claim that, for each $\lambda \in(0,1)$ and $\varepsilon>0$,

$$
\lambda p+(1-\lambda) q+\varepsilon B \subseteq \lambda p+(1-\lambda) S+\varepsilon B .
$$

\footnotetext{
${ }^{15}$ We note that the significance of the set $\mathscr{C}(\succsim)$ for expected utility theory without the completeness axiom was observed first by Aumann [2]. Like that of Aumann, the primary element of the approach we adopt here is the investigation of the geometry of $\mathscr{C}(\succsim)$. This approach is also adopted by a number of authors in the literature, among which are $[3,8,11,24,25]$.
} 
To see this, for any $b \in B$, pick a $\delta>0$ small enough that $\varepsilon b+\delta B \subseteq \varepsilon B$. Since $q$ belongs to the boundary of $S$ (in aff $(S)$ ), there must exist a $\omega \in S$ with $\omega \in q+\delta B$. But then $(1-\lambda)(q-w)+\varepsilon b \in \delta B+\varepsilon b \subseteq \varepsilon B$ so that

$$
\begin{aligned}
\lambda p+(1-\lambda) q+\varepsilon b & =\lambda p+(1-\lambda)(q+w-w)+\varepsilon b \\
& =\lambda p+(1-\lambda) w+(1-\lambda)(q-w)+\varepsilon b \\
& \subseteq \lambda p+(1-\lambda) S+\varepsilon B .
\end{aligned}
$$

Now, since $p$ is in the relative interior of $S$, for any $\lambda \in(0,1)$ there exists an $\varepsilon>0$ small enough that $p+\frac{\varepsilon}{\lambda} B \subseteq S$ so that, by (4), we get

$$
\begin{aligned}
\lambda p+(1-\lambda) q+\varepsilon B & \subseteq \lambda p+(1-\lambda) S+\varepsilon B \\
& =\lambda\left(p+\frac{\varepsilon}{\lambda} B\right)+(1-\lambda) S \\
& \subseteq \lambda S+(1-\lambda) S \\
& =S .
\end{aligned}
$$

Since $0 \in B$, this proves that $\lambda p+(1-\lambda) q \in S$ for all $\lambda \in(0,1)$, so it follows from the weak continuity axiom that $q \in S$. Thus we may conclude that $S$ contains its boundary in $\operatorname{aff}(S)$, so it is closed in $\operatorname{aff}(S)$. But since aff $(S)$ is finite-dimensional, the closures of $S$ in $\operatorname{aff}(S)$ and $\mathscr{P}(X)$ are identical, so it follows that $S$ is closed in $\mathscr{P}(X)$.

To prove the expected multi-utility theorem, we need to introduce some terminology. In what follows, for any metric space $X$, we let ca $(X)$ stand for the set of all finite Borel signed measures on $X$. Clearly, ca $(X)$ is the linear space generated by $\mathscr{P}(X)$, that is,

$$
\operatorname{ca}(X):=\operatorname{span}(\mathscr{P}(X)) .
$$

It is well known that when $X$ is compact, $\mathrm{ca}(X)$ (normed by the total variation norm) is isometrically isomorphic to the topological dual of $C(X)$ (normed by the supnorm). Using this duality, we shall consider ca $(X)$ in this paper as endowed with the weak $^{*}$-topology. ${ }^{16}$ It is important to note that this weak ${ }^{*}$-topology on ca $(X)$ induces on the set of lotteries $\mathscr{P}(X)$ the standard topology of weak convergence for probability measures.

Proof of the expected multi-utility theorem. The necessity of the axioms for the representation is easy to verify; we shall rather focus here on their sufficiency. Let $\succsim$ satisfy the independence and continuity axioms, define $\mathscr{C}(\succsim)$ as in Lemma 2 , and recall that $\mathscr{C}(\gtrsim)$ is a convex cone in $\operatorname{ca}(X)$ such that $p \succsim q$ if and only if $p-$ $q \in \mathscr{C}(\gtrsim)$. The following claim provides the key step of the proof.

\footnotetext{
${ }^{16}$ Under this topology, a net $\left(\mu_{\alpha}\right)$ in ca $(X)$ converges to $\mu \in \mathrm{ca}(X)$ if and only if $\int_{X} f d \mu_{\alpha} \rightarrow \int_{X} f d \mu$ for all $f \in C(X)$.
} 
Claim 1. $\mathscr{C}(\gtrsim)$ is weak*-closed.

Proof of Claim 1. We shall first show that $\mathscr{C}(\gtrsim)$ is sequentially weak*-closed. Take then a sequence $\left(\lambda_{n}\left(p_{n}-q_{n}\right)\right)$ in $\mathscr{C}(\succsim)$, and assume that $\left(\lambda_{n}\left(p_{n}-q_{n}\right)\right)$ converges in $\mathrm{ca}(X)$ in the weak ${ }^{*}$-topology. If $p_{n}=q_{n}$ for infinitely many $n$, then $\lambda_{n}\left(p_{n}-\right.$ $\left.q_{n}\right) \rightarrow 0 \in \mathscr{C}(\gtrsim)$, hence it is without loss of generality to assume that $p_{n} \neq q_{n}$ for each $n$. Now note that, by definition, $\int_{X} f d\left(\lambda_{n}\left(p_{n}-q_{n}\right)\right)$ must be a convergent real sequence for all $f \in C(X)$, and this implies that

$$
\sup \left\{\int_{X} f d\left(\lambda_{n}\left(p_{n}-q_{n}\right)\right): n=1,2, \ldots\right\}<\infty .
$$

By the Banach-Steinhaus theorem, therefore, there exists a real number $K$ such that

$$
\left\|\lambda_{n}\left(p_{n}-q_{n}\right)\right\| \leqslant K, \quad n=1,2, \ldots .
$$

Now, by using the Jordan decomposition theorem, we can write $p_{n}-q_{n}=\gamma_{n}\left(r_{n}-\right.$ $w_{n}$ ) for two mutually singular $r_{n}, w_{n} \in \mathscr{P}(X)$ such that $r_{n} \succsim w_{n}$ and $\gamma_{n}>0$. By mutual singularity, $\left\|r_{n}-w_{n}\right\|=2$. But then

$$
\left\|\lambda_{n}\left(p_{n}-q_{n}\right)\right\|=\left\|\lambda_{n} \gamma_{n}\left(r_{n}-w_{n}\right)\right\|=\lambda_{n} \gamma_{n}\left\|\left(r_{n}-w_{n}\right)\right\|=2 \lambda_{n} \gamma_{n}
$$

so that, by (5), we may conclude that $\left(\lambda_{n} \gamma_{n}\right)$ is a real sequence that lies in the closed interval $[0, K / 2]$. This sequence must then have a convergent subsequence $\left(\lambda_{n_{k}} \gamma_{n_{k}}\right)$. But since $X$ is compact, $\mathscr{P}(X)$ is a weak ${ }^{*}$-compact set in ca $(X)$, and hence both $\left(r_{n_{k}}\right)$ and $\left(w_{n_{k}}\right)$ must have (weak ${ }^{*}$-)convergent subsequences. ${ }^{17}$ Passing to these subsequences consecutively, we end up with convergent subsequences $\left(\lambda_{n_{k_{t}}} \gamma_{n_{k_{t}}}\right)$, $\left(r_{n_{k_{t}}}\right)$, and $\left(w_{n_{k_{t}}}\right)$. Let us write $\lambda_{n_{k_{t}}} \gamma_{n_{k_{t}}} \rightarrow \lambda, r_{n_{k_{t}}} \rightarrow p$ and $w_{n_{k_{t}}} \rightarrow q$ as $t \rightarrow \infty$. By continuity of $\succsim$, we have $p \succsim q$. Moreover,

$$
\lambda_{n_{k_{t}}}\left(p_{n_{k_{t}}}-q_{n_{k_{t}}}\right)=\left(\lambda_{n_{k_{t}}} \gamma_{n_{k_{t}}}\right)\left(r_{n_{k_{t}}}-w_{n_{k_{t}}}\right) \rightarrow \lambda(p-q)
$$

as $t \rightarrow \infty$. Since every subsequence of a convergent sequence converges to the limit of the mother sequence, we must then have $\lim \lambda_{n}\left(p_{n}-q_{n}\right)=\lambda(p-q) \in \mathscr{C}(\succsim)$, and hence we may conclude that $\mathscr{C}(\gtrsim)$ is sequentially weak ${ }^{*}$-closed.

Since $X$ is compact, $C(X)$ is separable, and ca $(X)$ is equal (i.e. isometrically isomorphic) to the topological dual of $C(X)$. But, by the Krein-ŠSmulian theorem, every sequentially weak ${ }^{*}$-closed convex set in the dual of a separable normed space is weak*-closed (cf. [17, Corollary 2.7.13]). Consequently, the previous observation implies that $\mathscr{C}(\gtrsim)$ is weak ${ }^{*}$-closed in $\mathrm{ca}(X)$.

\footnotetext{
${ }^{17}$ Since the weak ${ }^{*}$-topology on $\mathscr{P}(X)$ is identical to the standard topology of weak convergence on $\mathscr{P}(X)$, weak* -compactness of $\mathscr{P}(X)$ is an immediate consequence of the Prohorov theorem. Alternatively, one can supply a nonprobabilistic proof by using Alaoglu's theorem.
} 
We are now prepared to prove the theorem. Define

$$
\mathscr{U}:=\left\{u \in C(X): \int_{X} u d \mu \geqslant 0 \text { for all } \mu \in \mathscr{C}(\succsim)\right\}
$$

which is a nonempty closed and convex cone in $C(X)$. If $p \succsim q$, then $p-q \in \mathscr{C}(\succsim)$ so that $\int_{X} u d p \geqslant \int_{X} u d q$ for all $u \in \mathscr{U}$. To establish the converse, take any $p^{\prime}$ and $q^{\prime}$ in $\mathscr{P}(X)$ with

$$
\int_{X} u d p^{\prime} \geqslant \int_{X} u d q^{\prime} \text { for all } u \in \mathscr{U}
$$

and assume that $p^{\prime} \succsim q^{\prime}$ does not hold. This means that the sets $\left\{p^{\prime}-q^{\prime}\right\}$ and $\mathscr{C}(\succsim)$ are disjoint. Since $\mathscr{C}(\succsim)$ is a weak ${ }^{*}$-closed convex cone, then, by the Hahn-Banach separation theorem, there exists a continuous linear functional $T$ on ca $(X)$ and a real $\alpha$ such that $T(\mu) \geqslant \alpha>T\left(p^{\prime}-q^{\prime}\right)$ for all $\mu \in \mathscr{C}(\succsim)$ (cf. [1, Theorem 5.58]). Since $0 \in \mathscr{C}(\succsim)$, we have $0=T(0) \geqslant \alpha$ so that $0>T\left(p^{\prime}-q^{\prime}\right)$. Moreover, since $\mathscr{C}(\gtrsim)$ is a cone, we have $m T(\mu)=T(m \mu) \geqslant \alpha$ for any $\mu \in \mathscr{C}(\succsim)$ and $m \in \mathbf{N}$. This implies that $T(\mu) \geqslant 0$ for all $\mu \in \mathscr{C}(\gtrsim) .{ }^{18}$ That is, $T(\mu) \geqslant 0>T\left(p^{\prime}-q^{\prime}\right)$ for all $\mu \in \mathscr{C}(\gtrsim)$. Since $T$ is linear and continuous in the weak ${ }^{*}$-topology, there exists a $v \in C(X)$ such that $T(\mu)=\int_{X} v d \mu$ for all $\mu \in \mathrm{ca}(X)$ (cf. [1, Theorem 5.83]). Thus, we have

$$
\int_{X} v d \mu \geqslant 0>\int_{X} v d\left(p^{\prime}-q^{\prime}\right) \text { for all } \mu \in \mathscr{C}(\gtrsim) .
$$

This means that $v \in \mathscr{U}$ and $\int_{X} v d p^{\prime}<\int_{X} v d q^{\prime}$, which is a contradiction.

Proof of the uniqueness theorem. Since the "if" part is trivial, we shall prove here only the "only if" part. Suppose that we can find a $v \in C_{b}(X)$ such that $v \in\langle\mathscr{V}\rangle\left\langle\langle\mathscr{U}\rangle\right.$. Endowing $C_{b}(X)$ with the weak topology, we may apply the separating hyperplane theorem to find a nonzero signed measure $\mu \in \mathrm{ca}(X)$ such that

$$
\int_{X} v d \mu>0 \geqslant \int_{X} u d \mu \text { for all } u \in\langle\mathscr{U}\rangle .
$$

The latter inequalities imply that $0 \geqslant \int_{X} \theta \mathbf{1}_{X} d \mu=\theta \mu(X)$ for all real $\theta$, and hence we have $\mu(X)=0$. Of course, we have $\mu=\mu^{+}-\mu^{-}$for some finite Borel measures $\mu^{+}$ and $\mu^{-}$on $X$. By the previous observation, $\mu^{+}(X)=\mu^{-}(X)=c \geqslant 0$. Since $c=0$ would imply that $\mu=0$, we must actually have $c>0$. Thus $p:=\mu^{+} / c$ and $q:=\mu^{-} / c$ belong to $\mathscr{P}(X)$. So, by (6), we get $\int_{X} v d p>\int_{X} v d q$ and $\int_{X} u d p \leqslant \int_{X} u d q$ for all $u \in\langle\mathscr{U}\rangle$, which is a contradiction.

\footnotetext{
${ }^{18}$ The last three sentences and the geometric form of the Hahn-Banach theorem show that a closed convex cone can be strictly separated from a point in its exterior by a closed hyperplane which passes through the origin. We shall use this form of the separating hyperplane theorem also in the uniqueness theorem that follows.
} 
We conclude with two technical remarks that intend to clarify the distinction between the arguments needed to prove the expected multi-utility theorem in the cases where $X$ is finite and infinite.

Remark 1. Much of the analysis above is based on the fact that applying the supporting hyperplane theorem to the convex cone $\mathscr{C}(\gtrsim)$ yields readily a set of weak $^{*}$-continuous linear functionals $T$ on ca $(X)$ that allows us represent $\succsim$ as in (3). But the use of the supporting hyperplane theorem is warranted only if $\mathscr{C}(\gtrsim)$ is weak*-closed in ca $(X)$. This is not a serious problem when $X$ is finite, for then $\operatorname{ca}(X)$ is none other then $\mathbf{R}^{|X|}$, and we can verify relatively easily that the (weak) continuity of $\succsim$ is enough to guarantee that $\mathscr{C}(\gtrsim)$ is closed; this is the gist of the proof of Proposition 1. Claim 1 above shows that if we strengthen weak continuity to the continuity axiom, we can again guarantee that $\mathscr{C}(\succsim)$ is closed in $\mathrm{ca}(X)$ when $X$ is an infinite (yet compact) set. This requires harder work, because ca $(X)$ is a locally convex topological linear space which is not metrizable when $|X|=\infty$. A natural question is if one can do away with these complications by focusing instead of $\mathscr{P}(X)$ on the set $\mathscr{P}_{S}(X)$ of all simple lotteries on $X$, and using the independence and continuity axioms only for lotteries that belong to $\mathscr{P}_{s}(X)$. Contrary to what one may at first expect, the answer is no (even when $X$ is compact), for we do not know if (and how) one can show that $\mathscr{C}(\succsim)$ is weak*-closed in the linear space $Y$ generated by $\mathscr{P}_{s}(X)$. In particular, the method adopted in Proposition 1 fails here because $Y$ is infinite-dimensional, and the idea behind Claim 1 does not apply because $Y$ is not a Banach space.

Remark 2. While our main representation theorem is strong enough to cover many cases of interest, it does not function in the general domain that the classical (topological version) of the expected utility theorem (that yields continuous utility functions over the prize space) functions, namely, for preferences defined over lotteries on an arbitrary separable metric space. Whether our result can be extended to this general domain is presently an open problem. It may be worth noting that the main difficulty in this regard is that, when $X$ is not compact, the "natural" topologies on $C_{b}(X)$ and $\mathrm{ca}(X)$ (induced by the dual pair structure $\left(C_{b}(X), \mathrm{ca}(X)\right)$ under the duality map $\left.(f, \mu) \mapsto \int_{X} f d \mu\right)$ differs from the standard weak and weak* topologies (induced by the sup-norm). This, in turn, invalidates the arguments given in the key step (Claim 1) of the proof of our main theorem; in particular, the KreinŚmulian theorem does not apply in this context.

\section{Acknowledgments}

We thank Kim Border, Erio Castagnoli, Federico Echenique, Marco LiCalzi, Massimo Marinacci, Tapan Mitra, Piero Muliere, Alfred Müller, Marco Scarsini, David Schmeidler, Srinivasa Varadhan and two referees of this journal for their valuable comments. Support from the C.V. Starr Center for Applied Economics at New York University, M.I.U.R. and Università Bocconi is also gratefully acknowledged. 


\section{References}

[1] C. Aliprantis, K. Border, Infinite Dimensional Analysis, Springer, Berlin, 1999.

[2] R. Aumann, Utility theory without the completeness axiom, Econometrica 30 (1962) 445-462.

[3] T. Bewley, Knightian uncertainty theory: Part I, Cowles Foundation Discussion Paper No. 807, 1986.

[4] E. Castagnoli, F. Maccheroni, Generalized stochastic dominance and unanimous preferences, in: G. Giorgi, F. Rossi (Eds.), Generalized Convexity and Optimization for Economic and Financial Decisions, Pitagora, Bologna, 1998.

[5] E. Danan, Behavioral foundations of incomplete preferences, Mimeo, Université de Paris I, 2001.

[6] J. Dubra, E.A. Ok, A model of procedural decision making in the presence of risk, Internat. Econ. Rev. 43 (2002) 1053-1080.

[7] K. Eliaz, E.A. Ok, Indifference or indecisiveness? Choice theoretic foundations of incomplete preferences, Mimeo, New York University, 2003.

[8] P. Fishburn, Separation theorems and expected utilities, J. Econ. Theory 11 (1975) 16-34.

[9] I. Gilboa, D. Schmeidler, Maxmin expected utility with non-unique prior, J. Math. Econ. 18 (1989) $141-153$.

[10] J-M. Grandmont, Continuity properties of a von Neumann-Morgenstern utility, J. Econ. Theory 4 (1972) 45-57.

[11] Y. Kannai, Existence of a utility in infinite dimensional partially ordered spaces, Israel J. Math. 1 (1963) 229-234.

[12] F. Maccheroni, Maxmin under risk, Econ. Theory 19 (2002) 823-831.

[13] M. Majumdar, A. Sen, A note on representing partial orderings, Rev. Econ. Stud. 43 (1976) 543-545.

[14] M. Mandler, Incomplete preferences and rational intransitivity of choice, Games Econ. Behav., forthcoming.

[15] Y. Masatlioglu, E.A. Ok, Rational choice with a status quo bias, Mimeo, New York University, 2002.

[16] A. Mas-Colell, M. Whinston, J. Green, Microeconomic Theory, Oxford University Press, New York, 1995.

[17] R. Megginson, An Introduction to Banach Space Theory, Springer, New York, 1998.

[18] K. Mosler, M. Scarsini, Stochastic Orders and Applications: A Classified Bibliography, Springer, New York, 1994.

[19] A. Müller, Stochastic orders generated by integrals: a unified study, Adv. Appl. Probab. 29 (1997) 414-428.

[20] K. Nehring, Rational choice and revealed preference without binariness, Social Choice Welfare 14 (1997) 403-425.

[21] E.A. Ok, Utility representation of an incomplete preference relation, J. Econ. Theory 104 (2002) 429-449.

[22] T. Seidenfeld, M. Schervish, J. Kadane, A representation of partially ordered preferences, Ann. Statist. 23 (1995) 2168-2217.

[23] M. Shaked, J.G. Shanthikumar, Stochastic Orders and Their Applications, Academic Press, London, 1994.

[24] L. Shapley, M. Baucells, Multiperson utility, UCLA Working Paper 779, 1998.

[25] K. Vind, von Neumann Morgenstern preferences, J. Math. Econ. 33 (2000) 109-122.

[26] J. von Neumann, O. Morgenstern, Theory of Games and Economic Behavior, Princeton University Press, Princeton, 1944.

[27] W. Whitt, Stochastic comparisons for non-Markov processes, Math. Oper. Res. 11 (1986) 608-618. 